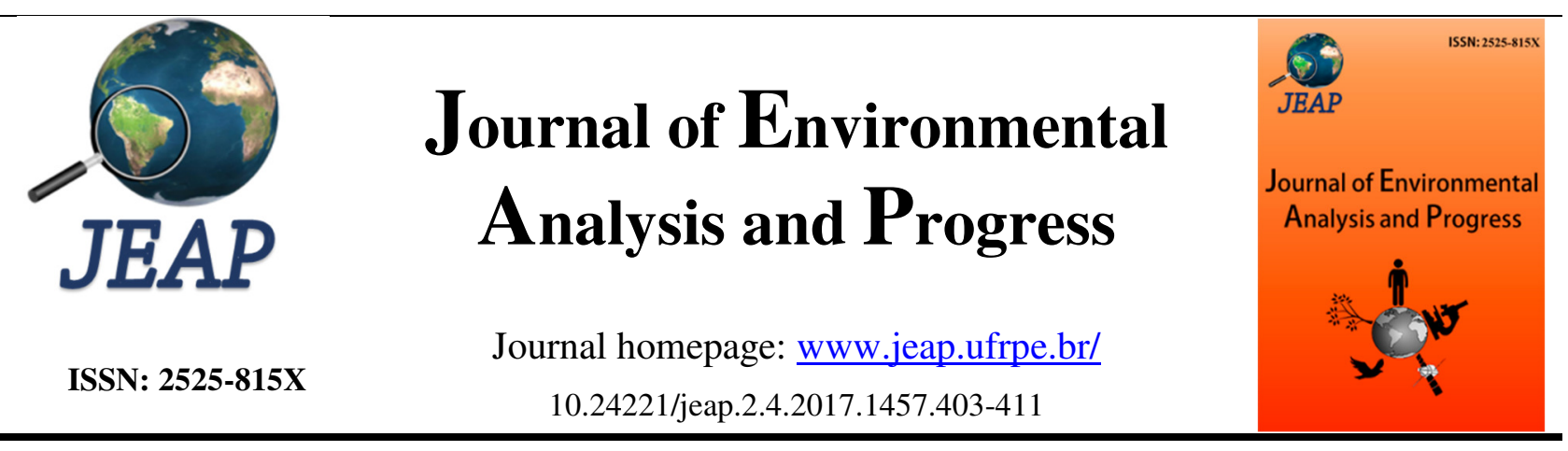

\title{
Influência dos elementos meteorológicos sobre a evapotranspiração de referência em Tauá, no Ceará
}

\section{Influence of meteorological elements on reference evapotranspiration in Tauá, in Ceará}

Joaquim Branco de Oliveira ${ }^{\mathrm{a}}$, Paula Juliana de Aquino Barbosa ${ }^{\mathrm{a}}$, Dijauma Honório Nogueira ${ }^{\mathrm{a}}$, Efraim Martins Araújo ${ }^{a}$, Francisco Dirceu Duarte Arraes ${ }^{b}$

a Instituto Federal de Educação, Ciência e Tecnologia do Ceará-IFCE, Laboratório de Geoprocessamento, Campus Iguatu, Rodovia Iguatu, Várzea Alegre, Km 05, s/n, Vila Cajazeiras, Iguatu, Ceará, Brasil. CEP: 63500-000. E-mail: joaquimbranco@ifce.edu.br; p.Juliana.barbosa345@gmail.com; dijaumahonorio@ifce.edu.br; efraimifce@gmail.com.

b IFCE Sertão, Campus Salgueiro, BR 232, Km 508, sentido Recife, Zona Rural, Salgueiro-PE, Brasil. CEP: 56.000-000. E-mail: dirceuarraes@gmail.com.

\section{A R T I C L E I N F O}

Recebido 30 Jun 2017

Aceito 01 Jul 2017

Publicado 15 Ago 2017

\begin{abstract}
A B S T R A C T
The reference evapotranspiration is an important agrometeorological parameter, mainly for irrigation planning and management and can be estimated by a wide variety of methods. In Brazilian Northeast, where rainfall is irregular, and water resources are usually contingent, the study of evapotranspiration is presented as a way of optimizing irrigation management. The study aimed to analyze the influence of climate elements on the reference evapotranspiration. It was calculated by the FAO-56 Penman-Monteith equation for the items of maximum and minimum temperature of air, relative humidity, wind speed and insolation for a period of 53, given by INMET, of the city of Tauá in Ceará, whose climate according to the classification of Köppen is BSw'h', hot and semi-arid climate. The reference evapotranspiration was calculated according to the procedures suggested by FAO 56 . The sensitivity analysis was based on the addition and decrease of one climactic element at a time in $\pm 5 \%$ up to the limit of $\pm 40 \%$ between $-40 \%$ and $-30 \%,-20 \%$, $-15 \%,-10 \%,-5 \%,+5 \%,+10 \%,+15 \%,+20 \%,+30 \%$ and $+40 \%$, while maintaining the values of the other elements Constant. The results showed the sensitivity of the model Penman-Monteith FAO 56 the variation of the elements of the climate in the rainy and dry period. Penman-Monteith, in the climatic conditions of Tauá, CE, is more sensitive to variations in maximum temperature and humidity of the air, followed by insolation, wind speed, and minimum air temperature.
\end{abstract}

Keywords: Penman-Monteith, sensitivity analysis, semi-arid.

\section{R E S U M O}

A evapotranspiração de referência é um importante parâmetro agrometeorológico, principalmente para planejamento e manejo de irrigação e pode ser estimada por uma ampla diversidade de métodos. No Nordeste brasileiro, onde as chuvas são irregulares e os recursos hídricos normalmente são contingenciados, o estudo da evapotranspiração se apresenta como uma forma de otimizar o manejo da irrigação. Este estudo objetivou analisar a influência dos elementos do clima na evapotranspiração de referência. Isto foi calculado pela equação de PenmanMonteith FAO-56. Os dados dos elementos do clima: temperatura máxima e mínima do ar, umidade relativa, velocidade do vento e insolação de um período de 53 anos, foram cedidos pelo INMET, da cidade de Tauá no Ceará, cujo clima segundo a classificação climática de Köppen é BSw'h', clima quente e semiárido. A 
evapotranspiração de referência foi calculada conforme os procedimentos sugeridos pela FAO 56. A análise de sensibilidade fundamentou-se no acréscimo e decréscimo de um elemento climático por vez em $\pm 5 \%$ até o limite de $\pm 40 \%$ dispostos entre $40 \%,-30 \%,-20 \%,-15 \%,-10 \%,-5 \%,+5 \%,+10 \%,+15 \%,+20 \%,+30 \%$ e $+40 \%$, mantendo-se os valores dos outros elementos constantes. Os resultados mostraram sensibilidade do modelo Penman-Monteith FAO 56 a variação dos elementos do clima nos períodos chuvoso e seco. Penman-Monteith, nas condições climáticas de Tauá, $\mathrm{CE}$, é mais sensível às variações da temperatura máxima e umidade do ar, seguido de insolação, velocidade do vento e temperatura mínima do ar.

Palavras-Chave: Penman-Monteith, análise de sensibilidade, semiárido.

\section{Introdução}

Atualmente nos deparamos, cada vez mais, com a escassez de água. Então, dizer que esse é um recurso renovável não se aplica mais, pois é fundamental para a sobrevivência da humanidade, importante para os mais diversos ramos da atividade humana.

A maior parte da água da superfície retorna à atmosfera pelos efeitos da evaporação e transpiração, sendo melhor percebida em ambientes onde estes dois processos ocorrem simultaneamente, pois em solos cobertos por vegetação, é na prática, impossível separar o vapor d'água proveniente da evaporação do solo daquele originado da transpiração das plantas. Nesse caso, um ambiente ideal é o semiárido.

Isso se dá porque a evaporação é responsável pelo movimento da água para o ar a partir de fontes como o solo, dossel florestal e corpos d'água, como lagos, córregos, rios e mares. A transpiração representa o movimento da água dentro de uma planta, e a consequente perda da mesma para a atmosfera (O Eco, 2014).

Diferentes ambientes apresentam diferentes taxas de evapotranspiração, uma vez que ela é afetada por vários fatores, como: estágio de crescimento da planta, crescimento ou nível de maturidade, fatores do solo e do clima.

Aperfeiçoar a gestão dos recursos hídricos é o primeiro passo para a conservação da água no nosso planeta. Nesse sentido, a evapotranspiração, como uma das componentes do balanço de água, é de grande utilidade em climatologia, para a quantificação das disponibilidades hídricas regionais (Cardoso et al., 1997).

Os métodos para a estimativa da ETo são geralmente dependentes de diversas variáveis atmosféricas, como o método de PenmanMonteith, parametrizado no boletim 56 da FAO, onde em muitas situações podem provocar dificuldades aos produtores rurais e pesquisadores que não possuem essas informações para a região, forçando-os a busca por outros métodos que não sejam tão dependentes de muitas variáveis.

Sabendo-se que o Nordeste brasileiro é caracterizado pelo clima semiárido no qual as chuvas são irregulares, o estudo da evapotranspiração se apresenta como uma oportunidade de se conhecer melhor esse contexto climático.

O objetivo deste estudo foi analisar a sensibilidade da equação de Penman-Montheith, parametrizada pela FAO, aos elementos do clima.

\section{Material e Métodos}

O estudo foi realizado na cidade de Tauá, no Estado do Ceará, localizada nos Inhamus no semiárido do sertão nordestino; as características da área de estudo estão descritas na Tabela 1.

Tabela 1. Dados geográficos e meteorológicos médios anuais da cidade de Tauá, no Ceará. IFCE, campus Iguatu, em 2017.

\begin{tabular}{cccccccccc}
\hline Local & $\boldsymbol{\varphi}$ & $\boldsymbol{\lambda}$ & $\mathbf{z}$ & $\mathbf{C c}$ & Chuva & Ta & $\mathbf{n}$ & UR & $\mathbf{u}_{\mathbf{1 0}}$ \\
\hline Tauá & $06^{\circ} 00^{\prime} 11^{\prime \prime} \mathrm{S}$ & $40^{\circ} 17^{\prime} 34^{\prime \prime} \mathrm{O}$ & $402 \mathrm{~m}$ & $\mathrm{BSw} \mathrm{h}^{\prime}$ & $715,8 \mathrm{~mm} \cdot \mathrm{ano}^{-1}$ & $25,3^{\circ} \mathrm{C}$ & $2515,0 \mathrm{~h}$ & $62,6 \%$ & $3,05 \mathrm{~m}^{-1} \mathrm{~s}^{1}$ \\
\hline
\end{tabular}

onde $\varphi=$ Latitude; $\lambda=$ longitude; $\mathrm{z}=$ altitude; $\mathrm{Cc}$ $=$ classificação climática de köppen; $\mathrm{Ta}=$ temperatura do ar média anual; $\mathrm{n}=$ insolação total anual; $\mathrm{UR}=$ umidade relativa média anual; $\mathrm{u} 10_{-}=$ velocidade do vento média anual a $10 \mathrm{~m}$ de altura.

Os dados do clima (Tabela 1), médias mensais de temperatura máxima, temperatura mínima, umidade relativa, velocidade do vento e insolação, usados no estudo foram obtidos na Estação Climatológica Principal pertencente ao
Instituto Nacional de Meteorologia (INMET). Foram calculadas médias mensais, utilizando um período de abrangência de 53 anos, de 1961 a 2014. $O$ preenchimento de falhas das séries foi realizado conforme os procedimentos sugeridos pelo FAO 56.

A velocidade do vento, medida a 10 metros da superfície, foi ajustada para a altura de 2 metros, conforme procedimento descrito por Allen et al. (1998). 
Para o cálculo da evapotranspiração e para a verificação dos possíveis efeitos das alterações dos elementos climáticos na evapotranspiração, foi utilizado o modelo parametrizado pela FAO (Allen et al., 1998).

$$
\mathrm{ET}_{\mathrm{o}}=\frac{(0,408) \Delta\left(\mathrm{R}_{\mathrm{n}}-\mathrm{G}\right)+\gamma \frac{900}{\mathrm{~T}+273} \mathrm{u}_{2}(\mathrm{es}-\mathrm{ea})}{\Delta+\gamma\left(1+0,34 \mathrm{u}_{2}\right)}
$$

onde: $\mathrm{ET}_{\mathrm{o}}=$ evapotranspiração de referência, em $m m . d^{-1} ; R n=$ saldo de radiação à superfície da cultura, em MJ.m. ${ }^{-2} \cdot \mathrm{d}^{-1} ; \mathrm{G}=$ densidade do fluxo de calor no solo, $\mathrm{MJ} \cdot \mathrm{m}^{-2} \cdot \mathrm{d}^{-1} ; \mathrm{T}=$ temperatura média do ar a $2 \mathrm{~m}$ de altura, $\mathrm{em}^{\circ} \mathrm{C} ; \mathrm{u}_{2}=$ velocidade do vento a $2 \mathrm{~m}$ de altura, em $\mathrm{m} \cdot \mathrm{s}^{-1}$; es $_{\text {s }}$ pressão de saturação de vapor, $\mathrm{kPa}$; e a pressão atual do vapor, $\mathrm{kPa} \Delta=$ declividade da curva de pressão de vapor, em $\mathrm{kPa} .{ }^{\circ} \mathrm{C}^{-1} ; \gamma=$ coeficiente psicométrico, $\mathrm{kPa}^{\circ} \mathrm{C}^{-1}$.

A declividade da curva de pressão de vapor é calculada em função da temperatura.

$\Delta=\frac{2504 \exp \left(\frac{17,27 \mathrm{~T}}{\mathrm{~T}+237,3}\right)}{(\mathrm{T}+237,3)^{2}}$

A temperatura média mensal $(\mathrm{T})$ é obtida em função da temperatura máxima $\left(\mathrm{T}_{\mathrm{x}}\right)$ e da temperatura mínima do ar $\left(\mathrm{T}_{\mathrm{n}}\right)$.

$\mathrm{T}=\frac{\mathrm{T}_{\mathrm{x}}+\mathrm{T}_{\mathrm{n}}}{2}$

$\mathrm{R}_{\mathrm{n}}=\mathrm{R}_{\mathrm{ns}}+\mathrm{R}_{\mathrm{nl}}$

$\mathrm{R}_{\mathrm{ns}}=(1-\alpha) \mathrm{R}_{\mathrm{s}}$

$\mathrm{R}_{\mathrm{nl}}=\sigma\left[\frac{\mathrm{T}_{\mathrm{x}} \mathrm{K}^{4}+\mathrm{T}_{\mathrm{n}} \mathrm{K}^{4}}{2}\right]\left(0,34-0,14 \sqrt{\mathrm{e}_{\mathrm{a}}}\right) \cdot\left(1,35 \frac{\mathrm{R}_{\mathrm{s}}}{\mathrm{R}_{\mathrm{so}}}-0,35\right)$

$\mathrm{R}_{\mathrm{s}}=\mathrm{R}_{\mathrm{a}}\left(0,25+0,50 \frac{\mathrm{n}}{\mathrm{N}}\right)$

$\mathrm{R}_{\mathrm{so}}=\left(0,75+2 \cdot 10^{-5} \mathrm{z}\right) \mathrm{R}_{\mathrm{a}}$

$\mathrm{R}_{\mathrm{a}}=\frac{24.60}{\pi} \mathrm{G}_{\mathrm{sc}} \mathrm{d}_{\mathrm{r}}\left[\omega_{\mathrm{s}} \operatorname{sen}(\varphi) \operatorname{sen}(\delta)+\cos (\omega) \cos (\delta) \operatorname{sen}\left(\omega_{\mathrm{s}}\right)\right]$

$\mathrm{e}_{\mathrm{a}}=\frac{\mathrm{UR}}{100}\left(\mathrm{e}_{\mathrm{s}}\right)$

$\mathrm{d}_{\mathrm{r}}=1+0,033 \cdot \cos \left(\frac{2 \pi}{365} \mathrm{~J}\right)$

$\delta=0,4093 \cdot \operatorname{sen}\left(\frac{2 \pi}{365} \mathrm{~J}-1,39\right)$

$\omega_{\mathrm{s}}=\cos ^{-1}(-\tan \varphi \cdot \tan \delta)$

$\mathrm{N}=\frac{24 \omega_{\mathrm{S}}}{\pi}$
A constante psicrométrica é calculada em função da pressão atmosférica.

$\gamma=0,00065 \times P$

A pressão atmosférica $(\mathrm{P})$ é dada em função da altitude (z) local.

$\mathrm{P}=101,3\left(\frac{293-0,0065 \mathrm{z}}{293}\right)^{5,26}$

A pressão de saturação do vapor $\left(\mathrm{e}_{\mathrm{s}}\right.$ ) é calculada em função da pressão de saturação da temperatura do ar $\left(\mathrm{C}^{\circ}\right)$ :

$\mathrm{e}_{\mathrm{s}}=\frac{\mathrm{e}_{\mathrm{s}}(\mathrm{Tx})+\mathrm{e}_{\mathrm{s}}(\mathrm{Tn})}{2}$

onde:

$e_{s}(T)=0,6108 \exp \left(\frac{17,27 T}{T+237,3}\right)$

Substituindo $\mathrm{T}$ na equação por $\mathrm{T}_{\mathrm{n}}$ obtemos a pressão de saturação da temperatura mínima do ar $\mathrm{e}_{\mathrm{s}}\left(\mathrm{T}_{\mathrm{n}}\right)$ e $\mathrm{T}$ por $\mathrm{T}_{\mathrm{x}}$ tem-se $\mathrm{e}_{\mathrm{S}}\left(\mathrm{T}_{\mathrm{x}}\right)$.

O saldo de radiação $\left(R_{n}\right)$ é a soma entre o saldo de radiação de onda curta $\left(R_{n s}\right)$ e o saldo de radiação de onda longa $\left(\mathrm{R}_{\mathrm{nl}}\right)$. OEles podem ser obtidos pelas Equações 8-18:

\section{Eq.(8)}

Eq.(9)

Eq.(10)

Eq.(11)

Eq.(12)

Eq.(13)

Eq.(14)

Eq.(15)

Eq.(16)

Eq.(17)

Eq.(18) 
onde $\mathrm{R}_{\mathrm{a}}$ = radiação solar no topo da atmosfera, em $M J \mathrm{~m}^{-2} \mathrm{~d}^{-1} ; \mathrm{R}_{\mathrm{s}}=$ radiação solar, $M J \mathrm{~m}^{-2} \mathrm{~d}^{-1} ; \mathrm{R}_{\mathrm{so}}=$ radiação solar global para dias de céu claro, em MJ $\mathrm{m}^{-2} \mathrm{~d}^{-1} ; \mathrm{n}=$ número de horas de brilho solar ou insolação, em horas; $\mathrm{N}=$ duração astronômica do dia, em horas; $\mathrm{e}_{\mathrm{a}}=$ pressão atual de vapor, em $\mathrm{kPa}$; $\sigma=$ constante de Stefan-Boltzmann, 4,903.10-9 , em $\mathrm{MJ} \cdot \mathrm{K}^{-4} \cdot \mathrm{m}^{-2} \cdot \mathrm{d}^{-1} ; \mathrm{T}_{\mathrm{x}} \mathrm{K}=$ temperatura máxima do ar, em $K ; T_{n} K=$ temperatura mínima do ar, em $K ; J=$ dia Juliano, em dias; $\delta=$ declinação solar, em radianos; $\mathrm{G}_{\mathrm{sc}}=$ constante solar, em 0,0820 ${\mathrm{MJ} . \mathrm{m}^{-}}^{-}$ ${ }^{2} \cdot \mathrm{min}^{-1} ; \quad \mathrm{d}_{\mathrm{r}}=$ distância relativa terra-sol, adimensional; $\omega_{\mathrm{s}}=$ ângulo horário do nascer ou pôr-do-sol, em radianos; $\varphi=$ latitude local, em radianos.

O fluxo de calor no solo $(\mathrm{G})$ é calculado em função temperatura do mês atual $\left(\mathrm{T}_{\mathrm{i}}\right)$ e a temperatura do mês anterior $\left(\mathrm{T}_{\mathrm{i}-1}\right)$ :

$$
\mathrm{G}=0,14\left(\mathrm{~T}_{\mathrm{i}}-\mathrm{T}_{\mathrm{i}-1}\right)
$$

A análise da sensibilidade fundamentouse no acréscimo e decréscimo dos valores dos elementos meteorológicos em alterações de até $40 \%$, um por vez e mantendo os valores dos outros elementos constantes, dispostos entre $-40 \%,-35 \%$, $-30 \%,-25 \%,-20 \%,-15 \%,-10 \%,-5 \%,+5 \%,+10 \%$, $+15 \%,+20 \%,+25 \%,+30 \%,+35 \%,+40 \%$.

Para quantificar o impacto de um intervalo de valores de um parâmetro de entrada sobre os resultados obtidos com o modelo, foram calculados coeficientes de sensibilidade para $T_{x}, T_{n}, n, U R, u_{2}$. Conforme procedimento sugerido por McCuen (1974):

$\mathrm{S}_{\mathrm{r}}=\frac{\Delta \mathrm{ET}_{0}}{\Delta \mathrm{V}_{\mathrm{i}}} \cdot \frac{\mathrm{V}_{\mathrm{i}}}{\mathrm{ET}_{0}}$

onde: $\mathrm{S}_{\mathrm{r}}=$ coeficiente de sensibilidade do modelo; $\Delta \mathrm{ET}_{\mathrm{o}}=$ variação na evapotranspiração de referência com a mudança no elemento meteorológico; $\Delta \mathrm{V}_{\mathrm{i}}=$ variação em cada parâmetro; $V_{\mathrm{i}}=$ parâmetro utilizado.

A variação do coeficiente de sensibilidade da $\mathrm{ET}_{\mathrm{o}}$ para série perturbada foi obtida conforme descrito por Gong et al. (2006):

$\frac{\Delta \mathrm{ET}_{0}}{\mathrm{ET}_{0}} \cong\left(\frac{\Delta \mathrm{T}_{\mathrm{x}}}{\mathrm{T}_{\mathrm{x}}}\right) \cdot \mathrm{S}_{\mathrm{r}}\left(\mathrm{T}_{\mathrm{x}}\right)+\left(\frac{\Delta \mathrm{T}_{\mathrm{n}}}{\mathrm{T}_{\mathrm{n}}}\right) \cdot \mathrm{S}_{\mathrm{r}}\left(\mathrm{T}_{\mathrm{n}}\right)+\left(\frac{\Delta \mathrm{n}}{\mathrm{n}}\right) \cdot \mathrm{S}_{\mathrm{r}}(\mathrm{n})+\left(\frac{\Delta \mathrm{UR}}{\mathrm{UR}}\right) \cdot \mathrm{S}_{\mathrm{r}}(\mathrm{UR})+\left(\frac{\Delta \mathrm{u}_{2}}{\mathrm{u}_{2}}\right) \cdot \mathrm{S}_{\mathrm{r}}\left(\mathrm{u}_{2}\right)$

onde: $\Delta \mathrm{T}_{\mathrm{x}}=$ variação na temperatura máxima do ar, em ${ }^{\circ} \mathrm{C} ; \mathrm{S}_{\mathrm{r}}\left(\mathrm{T}_{\mathrm{x}}\right)=$ coeficiente de sensibilidade para temperatura máxima do ar; $\Delta \mathrm{T}_{\mathrm{n}}=$ variação na temperatura mínima, em ${ }^{\circ} \mathrm{C} ; \mathrm{S}_{\mathrm{r}}\left(\mathrm{T}_{\mathrm{n}}\right)=$ coeficiente de sensibilidade para temperatura mínima do ar; $\Delta \mathrm{n}=$ variação na insolação, em horas; $S_{\mathrm{r}}(\mathrm{n})=$ coeficiente de sensibilidade para insolação; $\Delta \mathrm{UR}=$ variação na umidade relativa do ar, em \%; $\mathrm{S}_{\mathrm{r}}(\mathrm{UR})=$ coeficiente de sensibilidade para umidade relativa do ar; $\Delta \mathrm{u}_{2}=$ variação na velocidade do vento a $2 \mathrm{~m}$ de altura, em $\mathrm{m} . \mathrm{s}^{-1} ; \mathrm{S}_{\mathrm{r}}\left(\mathrm{u}_{2}\right)=$ coeficiente de sensibilidade para velocidade do vento a $2 \mathrm{~m}$ de altura.

\section{Resultados}

Com a perturbação da série de elementos do clima em 5\% praticamente não ocorreu alteração na variação da evapotranspiração. A partir de $10 \%$ de perturbação nos elementos do clima, observou-se comportamento diferenciado da evapotranspiração ao longo do ano. Especialmente entre os períodos de janeiro a junho e de julho a dezembro (Figura 1).

De julho a dezembro, período mais quente e seco do ano, a variação dos elementos do clima tem maior influência na Equação de PenmanMonteith, no período chuvoso (fevereiro a maio), ao contrário, a influência dos elementos do clima na evapotranspiração é menor (Figura 1).
Em geral, a medida em que se eleva a magnitude dos incrementos e decrementos dos elementos do clima, a variação na ETo segue a mesma tendência, tendo maior destaque as alterações de $\pm 40 \%$. As linhas que indicam variações na ETo são bastante pronunciadas. Essa diferença é causada pela influência da temperatura do ar bem diferenciada entre o período seco e chuvoso (Figura 1).

Enquanto os incrementos nos elementos climáticos de temperatura máxima e mínima do ar, insolação e velocidade do vento provocam aumento na ETo, a umidade relativa observou-se o contrário. Com o aumento da umidade relativa diminui a ETo.

A ETo se mostrou mais sensível aos elementos do clima da temperatura máxima e umidade relativa do ar, e para os elementos de insolação e velocidade do vento. A temperatura mínima do ar foi o elemento climático com menor influência na ETo (Figura 2).

Enquanto os coeficientes de sensibilidade da evapotranspiração foram menores com o decremento e maiores com o incremento nos elementos do clima, a umidade relativa do ar apresentou comportamento diferente, o coeficiente de sensibilidade foi maior no decremento e menor no incremento (Figura 2). 
Os resultados apontaram que o saldo de radiação é a variável mais sensitiva no cálculo do balanço de energia, enquanto o fluxo de calor no solo oferece a menor contribuição. Os erros relativos de todas as variáveis envolvidas no cálculo do balanço de energia baseado na razão de
Bowen aumentaram, significativamente, com o aumento do intervalo de amostragem.

A variável mais sensível na determinação da $\mathrm{ET}_{\mathrm{o}}$ pelo método de Penman-Monteith é o saldo de radiação seguida da umidade relativa, velocidade do vento a $2 \mathrm{~m}$ de altura e a temperatura média do ar.

Figura 1. Variação média da $\Delta$ ETo para os incrementos e decrementos de $\pm 5 \%$ (a), $\pm 10 \%$ (b), $\pm 15 \%$ (c), \pm $20 \%$ (d), $\pm 25 \%$ (e), $\pm 30 \%$ (f), $\pm 35 \%$ (g), e $\pm 40 \%$ (h), dos elementos meteorológicos para a cidade de TauáCE.
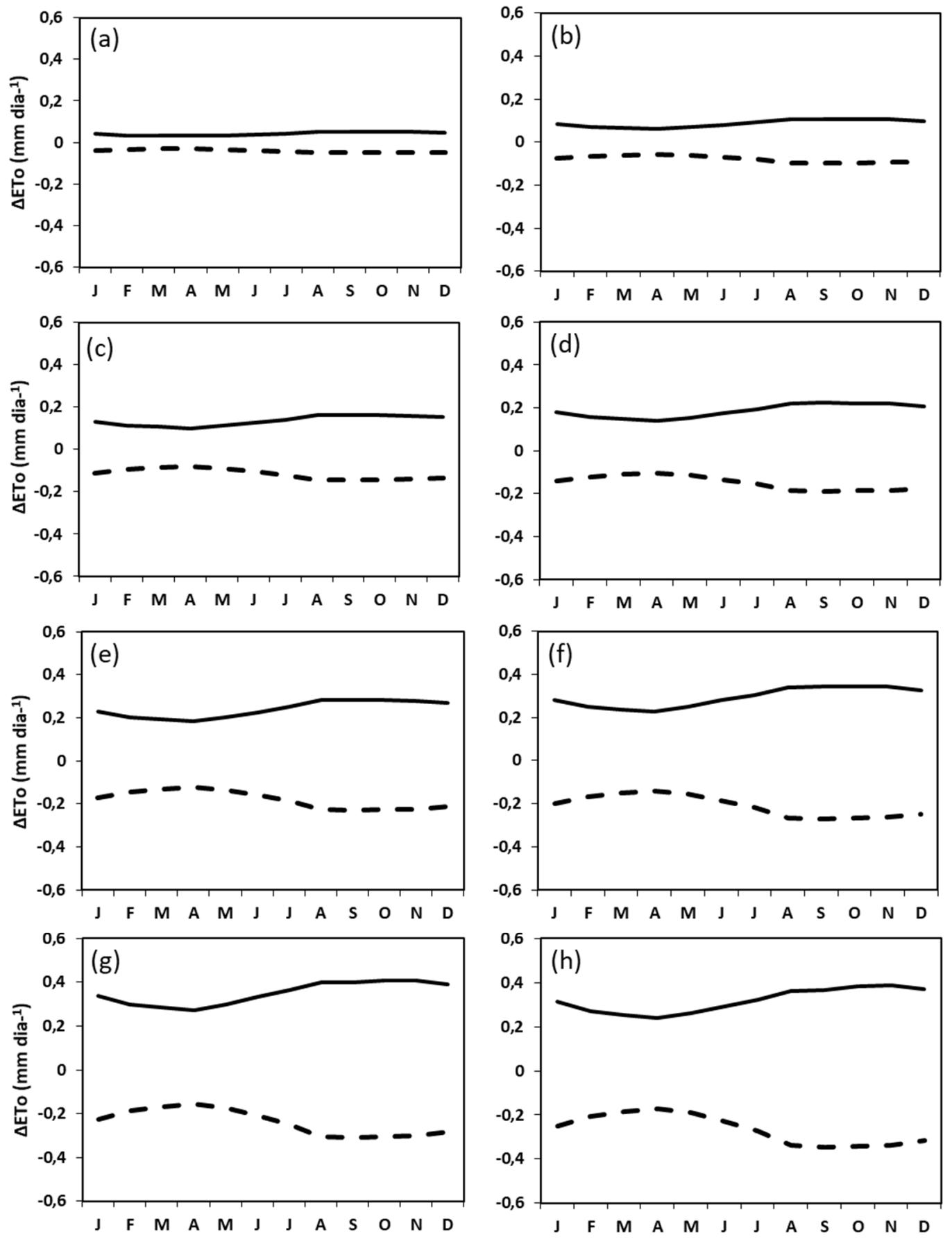

Comparativamente, fica evidente que na análise de sensibilidade quanto mais distantes de zero maior a sensibilidade da evapotranspiração ao elemento climático e quanto mais próximos a zero

menor o impacto do elemento climático na evapotranspiração (Figura 3). O motivo para a determinação dos coeficientes com base mensal foi analisar as modificações mensais desses 
coeficientes, para melhor entendimento do efeito relativo de cada elemento meteorológico sobre a ETo.
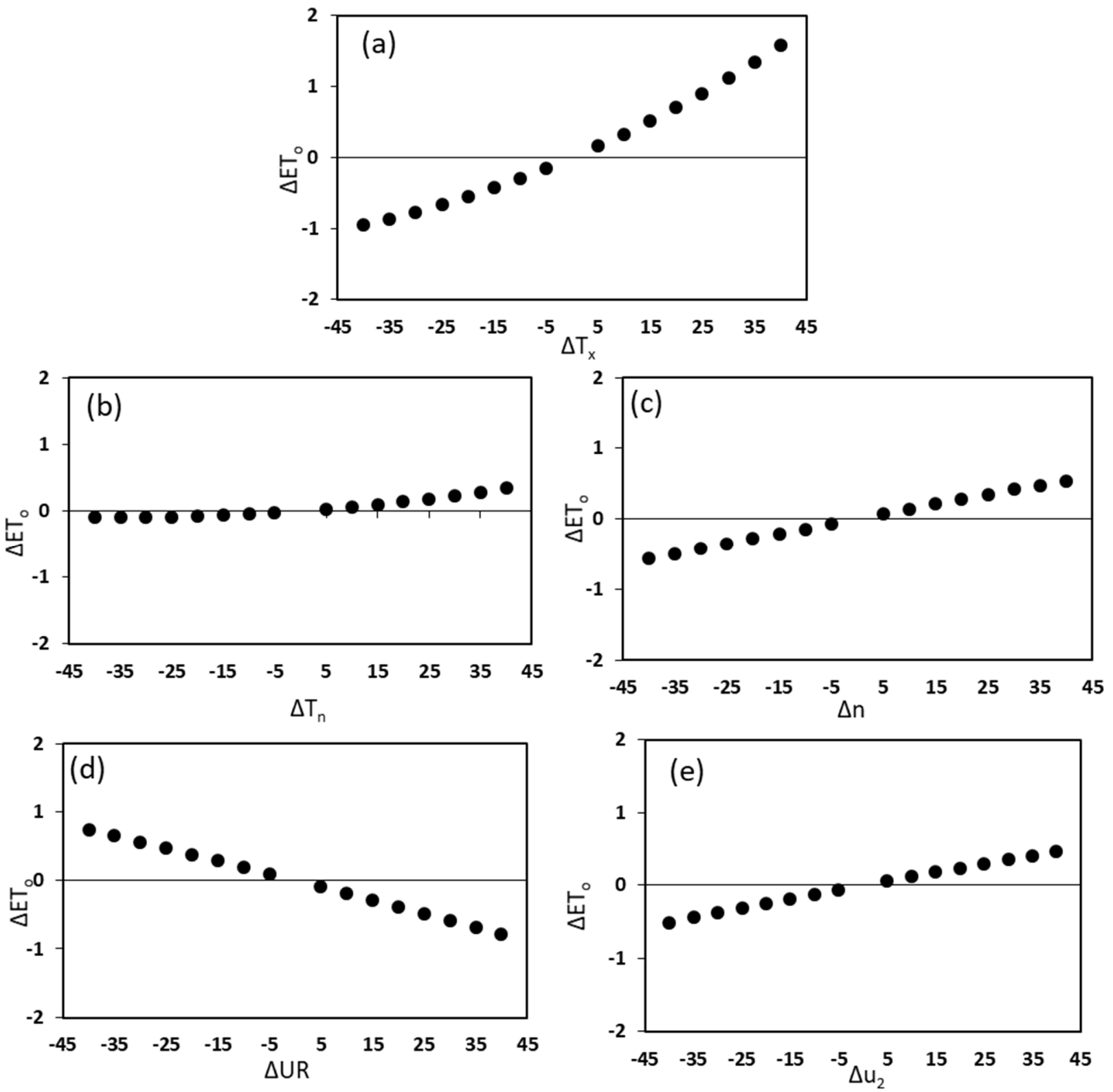

Figura 2. Variação da evapotranspiração de referência (ETo) em função da temperatura máxima do ar (a), temperatura mínima do ar (b), insolação (c), umidade relativa (d) e velocidade do vento (e) na cidade de TauáCE.

De modo geral, os coeficientes de sensibilidade para temperatura máxima do ar foram os que mostraram maior coeficiente de sensibilidade com 0,62 , seguido da umidade relativa com $-0,39$, insolação com 0,28 , velocidade do vento com 0,23 e temperatura mínima do ar com 0,11 (Figura 3).

Os coeficientes de sensibilidade calculados mostraram oscilações mensais sazonais para todos os elementos meteorológicos.
Pode-se notar, que os elementos de insolação e temperatura mínima do ar mostraram comportamentos semelhantes, no período chuvoso de fevereiro a maio, com os coeficientes de sensibilidade maiores que no período seco. A velocidade do vento mostrou maiores coeficientes de sensibilidade no período seco de julho a dezembro do que no período chuvoso (Figura 3). 


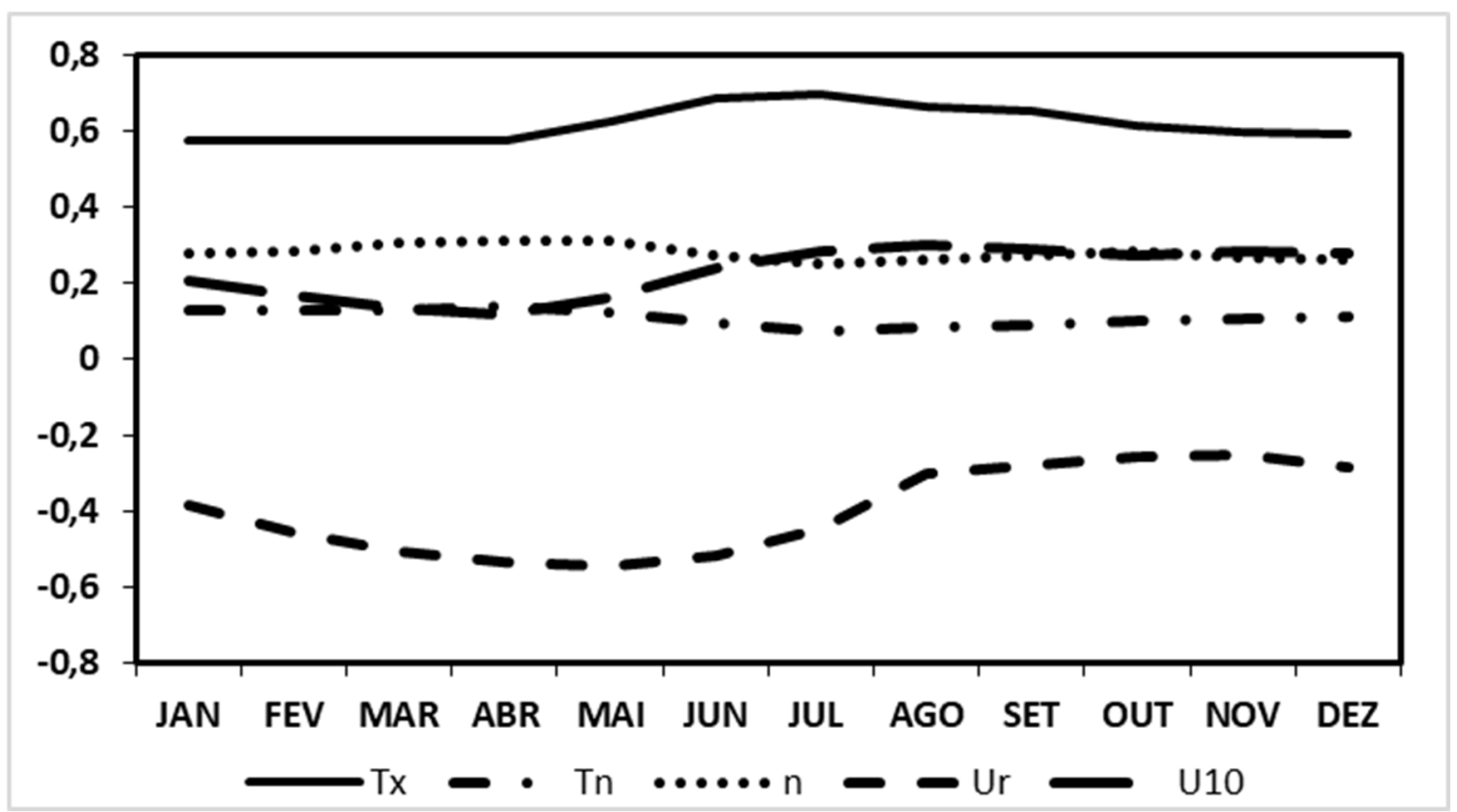

Figura 3. Variação média mensal dos coeficientes de sensibilidade de temperatura máxima (Tx) do ar, temperatura mínima (Tn) do ar, insolação (n), umidade relativa (Ur) do ar e velocidade do vento (U10), em Tauá-CE.

Há uma grande sensibilidade da ETo à temperatura máxima e à umidade relativa do ar, sendo que a temperatura máxima tem maior influência na ETo nos meses de junho e julho do que nos demais meses do ano. O comportamento da sensibilidade da ETo em relação à umidade relativa foi bem diferenciado, no período seco de agosto a dezembro, e no chuvoso de fevereiro a junho; no primeiro, a ETo é menos sensível à umidade relativa, enquanto no período chuvoso é mais sensível à umidade relativa (Figura 3).

Resultados semelhantes foram encontrados por Oliveira et al. (2016), analisando a sensibilidade dos elementos meteorológicos na equação de Penman-Monteith na cidade de Iguatu no Ceará, onde na estação chuvosa a influência dos elementos do clima na evapotranspiração de referência foi menos intensa que no período seco de junho a dezembro.

\section{Discussão}

Em estudo realizado por Silva et al. (2011), em experimento de campo conduzido no ano de 2009, na Destilaria Miriri, localizada no município de Capim, PB, com os dados de evapotranspiração de referência $\left(\mathrm{ET}_{\mathrm{o}}\right)$, obtidos pela equação de Penman-Monteith FAO/56, e da evapotranspiração da cultura da cana-de-açúcar $\left(\mathrm{ET}_{\mathrm{c}}\right)$, obtidos pelo balanço de energia com base na razão de Bowen, foram encontrados os maiores valores da evapotranspiração nos períodos de setembro de 2008, fevereiro, julho e setembro de 2009, com valores superiores a $7,0 \mathrm{~mm} \mathrm{~d}^{-1}$, período que corresponde à época de alta demanda atmosférica e também de maior disponibilidade de água no solo através da precipitação ou irrigação.

Segundo Silva et al. (2011), os valores dos coeficientes de sensibilidade das variáveis envolvidas no cálculo da evapotranspiração de referência variaram, consideravelmente, durante o período de estudo. No curso anual dos valores dos coeficientes de sensibilidade do saldo de radiação $\left(\mathrm{S}_{\mathrm{Rn}}\right)$, temperatura do $\operatorname{ar}\left(\mathrm{S}_{\mathrm{T}}\right)$, umidade relativa $\left(\mathrm{S}_{\mathrm{E}}\right)$ e velocidade do vento $\left(\mathrm{S}_{\mathrm{U}}\right)$ observaram que os maiores valores do coeficiente de sensibilidade são para o saldo de radiação, que variaram de 0,19 a 0,97 . Por outro lado, os valores de $S_{\mathrm{E}}$ variaram de 0,03 a 0,81 e de $S_{U}$ entre $-0,22$ e 0,6 , no final de setembro de 2009. Os autores afirmam que o coeficiente $S_{\mathrm{T}}$ apresentou variação negativa, ou seja, ele foi o que menos contribuiu para o aumento da sensibilidade na estimativa de $\mathrm{ET}_{\mathrm{o}}$ no período estudado, com valores entre -1,4 e -0,04 ao longo de todo o período. Esses resultados se aproximam daqueles obtidos por Hupet \& Vanclooster (2001), ao evidenciarem que a radiação solar é a variável atmosférica que mais influencia o cálculo da $\mathrm{ET}_{\text {o }}$.

Por outro lado, Xu et al. (2006) observaram que a variável mais sensível a essa equação na sua área de estudo foi a umidade relativa do ar seguida de radiação de ondas curtas e temperatura do ar; a velocidade do vento apresentou os menores valores de coeficientes de sensibilidade, que foram explicados pela sua grande variabilidade espacial na região.

No estudo realizado na Destilaria Miriri, localizada no município de Capim, PB, as análises de sensibilidade e de erros foram aplicadas ao 
método da razão de Bowen para os intervalos de medição de 15, 30, 60 e 120 minutos, enquanto o método de Penman-Monteith foi aplicado para a escala diária durante o ano de 2009.

Os resultados encontrados para a cidade de Iguatu no Ceará foram bastante semelhantes, onde o modelo de Penman-Monteith se mostrou mais sensível aos elementos de temperatura máxima do ar e umidade relativa (Oliveira et al., 2016).

Resultados semelhantes foram encontrados por Alencar, Sediyama \& Wanderley (2012) em estudo da Evolução temporal da evapotranspiração de referência e sua sensibilidade aos principais elementos climáticos na cidade de Uberaba-MG, onde pode-se notar que as duas variáveis mais sensíveis foram temperatura máxima e umidade relativa, seguidos de insolação, velocidade do vento e temperatura mínima, com os seguintes coeficientes de sensibilidade média: 1,$01 ;-0,53$; 0,$31 ; 0,19$; e $-0,15$, respectivamente.

Segundo os estudos de Carvalho \& Reis Júnior (2008), as estimativas de evapotranspiração diferem entre si principalmente com relação à variabilidade diária, verificando-se que os modelos mais complexos, Penman e Penman-Monteith, e até mesmo o de Priestley-Taylor, apresentam uma variabilidade diária bastante expressiva, enquanto os modelos de Thornthwaite, Hargreaves \& Oudin, que são baseados, principalmente, na temperatura, tendem a refletir apenas a sazonalidade intra-anual, com taxas de ETo mais elevadas no período de primavera-verão. Embora não tenham sido mostrados, os modelos baseados na temperatura tendem a superestimar a ETo em dias de chuva, onde a radiação não é tão intensa e a umidade do ar é mais alta, ambas as variáveis não são utilizadas nestes modelos.

O estudo de Alencar, Sediyama \& Wanderley (2012) mostrou que a ETo mensal é superior a $90 \mathrm{~mm}$, em média, para todos os meses do ano, atingindo seu ponto máximo em outubro, com um valor médio de $152 \mathrm{~mm}$. A soma da evapotranspiração do período de setembro a março (período de maiores índices pluviométricos na região da pesquisa) correspondeu a $64 \%$ da evapotranspiração total anual que é de $1495 \mathrm{~mm}$ por ano. Os resultados mostraram-se coerentes com os resultados obtidos por Minuzzi, Vianello \& Sediyama (2010), estudando as oscilações climáticas para várias cidades de Minas Gerais. Eles verificaram que não houve tendências significativas para as temperaturas mínimas e máximas para a cidade de Uberaba, durante o período de 1961 a 2004.

\section{Conclusão}

O modelo de Penman-Monteith FAO 56 apresentou maior sensibilidade à temperatura máxima do ar e umidade relativa do ar, seguidos da insolação, velocidade do vento e temperatura mínima do ar.

No período chuvoso, a sensibilidade do modelo de Penman-Monteith FAO 56 às alterações dos elementos do clima é menor do que nos meses mais secos do ano.

\section{Agradecimentos}

Ao Laboratório de Geoprocessamento e ao Instituto Federal de Educação Ciências e Tecnologia do Ceará, Campus Iguatu, pelo apoio no desenvolvimento da pesquisa. Ao Instituto Nacional de Meterologia pela concessão dos dados.

\section{Referências}

ALENCAR, L. P.; SEDIYAMA， G. C.; WANDERLEY, H. S.; HENDERSON, S.; ALMEIDA, T. S.; DELGADO, R. C.; VIEIRA, G. H. S. 2012. Evolução temporal da evapotranspiração de referência e sua sensibilidade aos principais elementos climáticos na cidade de Uberaba-MG. Revista Brasileira de Ciências Agrárias, v. 7, n. 1, p. 159-166.

ALLEN R. G.; PEREIRA, L. S.; RAES, D. SMITH, M. 1998. Crop evapotranspirationguidelines. Rome: FAO. (FAO Irrigation and Drainage Paper, 56). 297p.

CARDOSO, M. J.; BASTOS, E. A.; ANDRADE JÚNIOR, A. S.; RODRIGUES, B. H. N. 1997. Uso da exigência térmica para a determinação do ciclo de cultivares de milho sob irrigação. In: X Cong. Bras. de Agrometeorologia. Anais... Piracicaba: SBAGRO, pp. 23-25.

CARVALHO, T. L. L.; REIS JÚNIOR, D. S. 2008. Avaliação da influência dos dados de evapotranspiração potencial no desempenho de modelos hidrológicos em bacias do semiárido cearense. IX Simpósio de Recursos Hídricos do Nordeste. 20p.

GONG, L.; XU, C.; CHEN, D.; HALLDIN, S.; CHEN, Y. D. 2006. Sensitivity of the PenmanMonteith reference evapotranspiration to key climatic variables in the Changjiang (Yangtze River) basin. Journal of Hidrology, v. 329, p. 620629.

HUPET, F.; VANCLOOSTER, M. 2001. Effect of the sampling frequency of meteorological variables on the estimation of the reference 
evapotranspiration. Journal of Hydrology, v. 243, n. 3, p. 192-204.

MCCUEN, R. H. 1974. A sensitivity and error analysis of procedures used for estimating evapotranspiration. Water Resources Bulletin, v. 10, n. 3, p. 486-498.

MINUZZI, R. B.; VIANELLO, R. L.; SEDIYAMA, G. C. 2010. Oscilações climáticas em Minas Gerais. Revista Brasileira de Meteorologia, v. 25, n. 2, p. 227-236.

O ECO. Dicionário ambiental. 2014. Disponível em: $\quad$ http://www.oeco.org.br/dicionarioambiental/28768-o-que-e-evapotranspiracao.

Acesso em: 11 de jan. 2017.

OLIVEIRA, J. B.; BRASIL, P. P.; ARRAES, F. D. D.; RODRIGUES, V. B.; OLIVEIRA, W. C.; CORDEIRO, A. Y. P. A. V. G. Análise de sensibilidade dos elementos meteorológicos na equação de Penman-Montieth FAO 56 no semiárido. In: Congresso Brasileiro de Meteorologia, 19., 2016, João Pessoa, Anais... João Pessoa: SBMET, 2016. Disponível em: www.cbmet2106.regg.co/anais\#. Acesso em: 05 abr. 2017.

SILVA, B. K. N.; SILVA, V. P. R.; AZEVEDO, P. V., FARIAS, C. H. A. 2011. Análise de sensibilidade dos métodos de estimativa da evapotranspiração de referência e razão de Bowen em cultura da cana-de-açúcar. Revista brasileira de engenharia agrícola e ambiental, Campina Grande, v. 15, n. 10, p. $1046-1053$.

XU, C., GONG, L., JIANG, T., CHEN, D., SINGH, V. P. 2006. Analysis of spatial distribution and temporal trend of reference evapotranspiration and pan evaporation in Changjiang (Yangtze River) catchment. Journal of Hydrology, v. 327, n. 1, p. 81-93. 